\title{
Bcl-2 promotes metastasis through the epithelial-to-mesenchymal transition in the BCap37 medullary breast cancer cell line
}

\author{
CHENGYONG DU ${ }^{1}$, XIAOCHEN ZHANG ${ }^{2}$, MINYA YAO $^{1}$, KEZHEN LV $^{1}$, JIANNAN WANG ${ }^{1}$, LUYAN CHEN $^{1}$, \\ YAOMIN CHEN $^{1}$, SHUQIAN WANG ${ }^{1}$ and PEIFEN FU ${ }^{1}$
}

Departments of ${ }^{1}$ Breast Surgery and ${ }^{2}$ Medical Oncology, The First Affiliated Hospital, College of Medicine, Zhejiang University, Hangzhou, Zhejiang 310003, P.R. China

Received June 6, 2017; Accepted January 3, 2018

DOI: $10.3892 / \mathrm{ol} .2018 .8455$

\begin{abstract}
Metastatic breast cancer is one of the major types of cancer in women. However, despite being the focus of considerable research efforts, its molecular mechanism remains to be fully elucidated. The B-cell lymphoma/leukemia gene-2 (Bcl-2) protein is well known for its role in inhibiting programmed cell death/apoptosis. However, little is known concerning its function in cell invasion and migration. In the present study, cell migration and invasion assays revealed that anti-apoptotic Bcl-2 protein induced migration and invasion without affecting cell proliferation in the BCap37 breast cancer cell line. In addition, it was found that the overexpression of Bcl-2 in BCap37 cells increased metastasis to the lung in a mouse model. Using western blotting and RT q-PCR analysis, it was demonstrated that the overexpression of Bcl-2 inhibited the expression of E-cadherin, an epithelial marker, whereas it increased the levels of mesenchymal markers $\mathrm{N}$-cadherin and vimentin. Therefore, the results suggested that Bcl-2 may induce cellular metastasis in breast cancer via the epithelial-to-mesenchymal transition.
\end{abstract}

\section{Introduction}

Breast cancer accounts for $25 \%$ of all cancer cases around the world. In 2012, breast cancer was reported to cause $15 \%$ of all cancer-associated mortality among women (1). Furthermore, cancer metastasis is the major cause of cancer-associated mortality. Metastatic breast cancer is one of the most life-threatening types of cancer in women, with a mortality rate of $>400,000$ each year worldwide (2). A series of molecular mechanisms are associated with the migration and invasion of breast cancer cells, including the upregulated

Correspondence to: Dr Peifen Fu, Department of Breast Surgery, The First Affiliated Hospital, College of Medicine, Zhejiang University, 79 Qingchun Road, Hangzhou, Zhejiang 310003, P.R. China E-mail: fupeifen@hotmail.com

Key words: breast cancer, B-cell lymphoma 2, metastasis, epithelial-to-mesenchymal transition expression of matrix metalloproteinases, the promotion of the epithelial-to-mesenchymal transition (EMT), and the activation of nuclear factor- $\kappa \mathrm{B}$, cancer-induced angiogenesis, vascular endothelial growth factor, and mammalian target of rapamycin signaling pathways $(3,4)$. However, cancer metastasis is a complex process and a detailed understanding of its mechanism requires further investigation.

B-cell lymphoma/leukemia gene-2 (Bcl-2) is the first proto-oncogene to be demonstrated to function by inhibiting programmed cell death/apoptosis (5). Bcl-2 integrates multiple survival- and death-related signals, which are generated within the cell (5-7). However, the mechanisms underlying the non-apoptotic functions of $\mathrm{Bcl}-2$ remain to be fully elucidated. Previously, Bcl-2 was found to be involved in cancer cell invasion and metastasis (8). At the cellular level, the overexpression of Bcl-2 was reported to increase invasion and migration in glioma $(9,10)$, lung $(11)$ and breast cancer $(12,13)$. In animal models, Kang et al showed that the overexpression of Bcl-2 mediated the metastasis of the MDA-MB-231 human mammary epithelial cell line to the bone (14). Bcl-2 has subsequently been shown to induce cellular metastasis to the lung with in mouse EpH4 mammary epithelial cell line (15). Efforts made to develop chemical inhibitors of BCL-2 have been reported extensively (16). In primary breast cancer, $75 \%$ of cases express elevated levels of Bcl-2, of which $85 \%$ are estrogen receptor (ER)-positive and $50 \%$ are human epidermal growth factor receptor 2 (HER-2)-positive tumors $(17,18)$. In breast cancer, there appears to be a correlation between a high level of Bcl-2 and a poorer clinical outcome $(19,20)$. The analysis of patient samples has shown that the expression of Bcl-2 in cancer cells was associated with liver metastasis in breast (21) and colorectal (22) cancer. Additionally, upregulation in the expression of $\mathrm{Bcl}-2$ was found during the progression from pre-invasive lesions to invasive carcinoma in lung cancer samples (11). The overexpression of Bcl-extra large (Bcl-XL), another member of the Bcl-2 family, induces invasion and metastasis (lymph node or distal metastasis) in patients with breast cancer (22). This evidence led the present study to focus on the function of Bcl-2 in the invasion and migration of breast cancer.

The EMT is one of the key events in cancer invasion and metastasis. It has been reported that reversing EMT may suppress the metastasis of breast cancer (23). The 
overexpression of $\mathrm{N}$-cadherin in $\mathrm{MCF}-7$ breast cancer cells also induces cell migration, invasion and metastasis, without altering the endogenous expression or adhesiveness of E-cadherin (24). Furthermore, it was reported that Bcl-2 regulated Twist-targeted genes, including the E-cadherin gene $(13,25)$. The overexpression of Bcl-2 may inhibit the expression of E-cadherin and promote EMT in different types of cell $(13,26,27)$. Therefore, $\mathrm{Bcl}-2$ may be involved directly or indirectly in the transcriptional regulation of EMT.

In the present study, it was shown that the overexpression of the anti-apoptotic protein $\mathrm{Bcl}-2$ increased the migration of BCap37 cells, an ER-negative, HER2-positive human medullary breast cancer cell line (28) and induced cancer metastasis in vivo. The results also indicated that this Bcl-2-induced metastasis may result from the progression of EMT.

\section{Materials and methods}

Mice and cell lines. Female Balb/c mice were purchased from Vital River Laboratories, Co., Ltd. (Beijing, China). The BCap37 human breast cancer cell line was purchased from the Cell Bank of the Chinese Scientific Academy (Shanghai, China). The BCap37 cells were cultured in Roswell Park Memorial Institute (RPMI)-1640 medium (cat. no. 31800105; Gibco; Thermo Fisher Scientific, Inc., Waltham, MA, USA) supplemented with $10 \%$ fetal bovine serum (FBS; cat. no. 04-0101-1; Biological Industries USA, Inc., Cromwell, CT, USA). The cell culture medium was replaced every 2-3 days, and cells were passaged using $0.25 \%$ trypsin-EDTA (cat. no. 25200056; Gibco; Thermo Fisher Scientific, Inc.), once they reached $90 \%$ confluence. The cultures were maintained at $37^{\circ} \mathrm{C}, 5 \% \mathrm{CO}_{2}$ in a water-jacketed incubator (Thermo Fisher Scientific, Inc.).

Knock down of Bcl-2 in BCap37 cells. The Bcl-2 short hairpin (sh)RNA 5'-CCGGCGTGATGAAGTACATACATTACTCGA GTAATGTATGTACTTCATCACGTTTTTG-3' (BC-shRNA-Bcl2 \#1) or 5'-CCGGGCACACCTGGATCCAGGATAACTCGA GTTATCCTGGATCCAGGTGTGCTTTTTG-3' (BC-shRNA-Bcl2 \#2) sequences were cloned into the pLKO.1-TRC cloning vector. The plasmids were transfected into 293T cells (Cell Bank of the Chinese Academy of Sciences, Beijing, China) using Lipofectamine 2000 (Invitrogen; Thermo Fisher Scientific, Inc.) and the supernatant containing lentiviral plasmids was collected to infect BCap37 cells. RNA silencing was analyzed using reverse transcription-quantitative polymerase chain reaction (RT-qPCR) and western blot analyses.

Vector construction, lentivirus production and cell transfection. A Bcl-2 overexpression vector was constructed by introducing the Bcl-2 coding sequence into the pCDH-CMV-EF1-Puro vector ( $\mathrm{pLVX}-\mathrm{Bcl} 2$ ). The $\mathrm{Bcl}-2$ cDNA sequence was amplified from BCap37 cDNA using the forward 5'-ATAGCTAGC GCCACCATGGCGCACGCTGGGAGAACA-3' and reverse 5'-ATAGCGGCCGCTCACTTGTGGCCCAGATAGGCAC-3' primers (the thermocycling steps are provided in Table I). The pLVX-Bcl2 vector was mixed with the psPAX2 and PMD2.G packaging plasmids, and cotransfected into the 293T cells using Lipofectamine 2000 (Invitrogen; Thermo Fisher Scientific, Inc.), according to the manufacturer's protocol. The virus particles were harvested $48 \mathrm{~h}$ following transfection. The BCap37 cells were infected with the harvested recombinant lentivirus and were isolated using puromycin to establish stable cell lines constitutively overexpressing Bcl-2 (BC-Bcl2). BCap37 cells transfected with an empty lentiviral vector served as a control (BC-Mock).

RNA isolation and RT-qPCR analysis. Total RNA was isolated using RNAiso Plus (Takara Biotechnology Co., Ltd., Dalian, China) and transcribed into cDNA using the PrimeScript RT Reagent kit (Takara Biotechnology Co., Ltd.). The RT-qPCR was performed using an ABI 7500 FAST Real-Time PCR system (Applied Biosystems; Thermo Fisher Scientific, Inc.) and a SYBR Green PCR kit (cat. no. RR420A, Takara Biotechnology Co., Ltd.). The thermocycling steps were according to the manufacturer's instruction and the qPCR primers were shown in Table II. The relative expression levels of mRNA were quantified with the $2^{-\Delta \Delta} \mathrm{Cq}$ method (29), following normalization using GAPDH endogenous reference.

Wound-healing assays. Following transfection, the cells were grown to $100 \%$ confluence in six-well plates. A micropipette tip was used to introduce a cross wound, and wound healing was observed $48 \mathrm{~h}$ later. Images were captured under phase-contrast microscopy (Olympus, Tokyo, Japan), immediately or $48 \mathrm{~h}$ following wounding.

Cell proliferation assay. Cell proliferation was analyzed using the Cell Counting kit-8 assay (CCK8; Dojindo Molecular Technologies, Inc., Kumamoto, Japan). The BCap37, BC-shRNA-NC, BC-shRNA-Bcl2, BC-Mock or BC-Bcl2 cells were seeded into 96-well plates at a density of 1,000 cells per well and the growth rates were subsequently evaluated. Each assay was performed in triplicate.

Cell migration and invasion assay. The cell migration and invasion assays were performed using Transwell chambers (EMD Millipore, Boston, MA, USA). For the invasion assay, the inserts were coated with Matrigel (BD Biosciences, Franklin Lakes, NJ, USA) on the upper surface. Following transfection, $6 \times 10^{4}$ BCap37 cells were suspended in $0.2 \mathrm{ml}$ serum-free medium and added to the inserts. Subsequently, $0.6 \mathrm{ml}$ RPMI-1640 medium supplemented with 10\% FBS was added to the lower compartment, as a chemoattractant. Following incubation at $37^{\circ} \mathrm{C}$ for $24 \mathrm{~h}$, the cells on the upper surface of the membrane were carefully removed using a cotton swab, and the cells on the lower surface were fixed with $100 \%$ methanol and stained with $0.1 \%$ crystal violet. Five visual fields (magnification, x200) of each insert were randomly selected and counted using light microscopy.

Western blot analysis. Cellular proteins were isolated in a protein extraction buffer (Beyotime Institute of Biotechnology, Haimen, China). Equal quantities ( $40 \mu \mathrm{g} /$ lane) of proteins were fractionated on 6-10\% SDS-PAGE gels and transferred onto polyvinylidene difluoride membranes. The membranes were incubated with anti-Bcl2 (1:1,000 dilution; cat. no. sc-130307), anti-E-cadherin (1:1,000 dilution; cat. no. sc-59780) and anti-vimentin (1:1,000 dilution; cat. no. sc-80975) primary antibodies (Santa Cruz Biotechnology, Inc., Santa Cruz, 
Table I. Thermocycling steps for B-cell lymphoma 2 gene amplification.

\begin{tabular}{lll}
\hline $98^{\circ} \mathrm{C}$ & $2 \mathrm{~min}$ (Ramp rate: $\left.4.4^{\circ} \mathrm{C} / \mathrm{sec}\right)$ & 1 cycle \\
$98^{\circ} \mathrm{C}$ & $10 \mathrm{sec}$ (Ramp rate: $\left.4.4^{\circ} \mathrm{C} / \mathrm{sec}\right)$ & 35 cycles \\
$55^{\circ} \mathrm{C}$ & $15 \mathrm{sec}$ (Ramp rate: $\left.2.2^{\circ} \mathrm{C} / \mathrm{sec}\right)$ & \\
$72^{\circ} \mathrm{C}$ & $15 \mathrm{sec}\left(\right.$ Ramp rate: $\left.2.2^{\circ} \mathrm{C} / \mathrm{sec}\right)$ & \\
$72^{\circ} \mathrm{C}$ & $10 \mathrm{~min}$ (Ramp rate: $\left.2.2^{\circ} \mathrm{C} / \mathrm{sec}\right)$ & 1 cycle \\
$4^{\circ} \mathrm{C}$ & $1 \mathrm{~min}$ (Ramp rate: $\left.2.2^{\circ} \mathrm{C} / \mathrm{sec}\right)$ & 1 cycle \\
\hline
\end{tabular}

Table II. Primers used for reverse transcription-quantitative polymerase chain reaction.

\begin{tabular}{ll}
\hline Primer & \multicolumn{1}{c}{ Sequence $\left(5^{\prime}-3^{\prime}\right)$} \\
\hline BCL-2-F & GTCTTCGCTGCGGAGATCAT \\
BCL-2-R & CATTCCGATATACGCTGGGAC \\
GAPDH-F & GGAGCGAGATCCCTCCAAAAT \\
GAPDH-R & GGCTGTTGTCATACTTCTCATGG
\end{tabular}

CA, USA) at $4^{\circ} \mathrm{C}$ overnight. Then, the blots were incubated for $2 \mathrm{~h}$ at room temperature in a 1:10,000 dilution of HRP goat anti-rabbit IgG antibody (cat. no. ab6721; Abcam, Cambridge, UK) after 3 washes with PBST. After extensive washing, antibody detection was accomplished with a sensitive substrate (Immun-Star ${ }^{\mathrm{TM}}$ Western $\mathrm{C}^{\mathrm{TM}}$ kit, 170-5070; Bio-Rad Laboratories, Inc., Hercules, CA, USA). $\beta$-actin (1:1,000 dilution; cat. no. ab20272; Abcam) was used for the normalization of protein loading. The image densitometry was performed using Image Lab software (version 5.2.1 Bio-Rad Laboratories).

Examination of tumor growth and metastasis in vivo. To develop human breast xenografts, in vitro grown BCap37, BC-shRNA-C, BC-shRNA-Bcl2, BC-Mock and BC-Bcl2 cells $\left(1 \times 10^{6}\right.$ cells in $\left.0.2 \mathrm{ml} \mathrm{PBS}\right)$ were implanted into the right flanks of homozygous female nude athymic mice (5-6 weeks old abd weighing 17-21 g). The mice were housed in a temperature-controlled environment with $12 \mathrm{~h}$ light and dark cycles and received a standard food and water diet ad libitum. Two perpendicular diameters (width and length) of the mouse tumors were measured three times a week until the animals were sacrificed after 30 days. In addition, a tumor growth curve was drawn based on the tumor volume and the corresponding time (days) post-injection. When the animals were sacrificed, the lungs were harvested and fixed in $10 \%$ neutral formalin and embedded in paraffin. $4 \mu \mathrm{m}$ sections were prepared and stained with hematoxylin and eosin (H\&E), followed by examination under a microscope and image capture. The area of metastasis (pixels) of each lung metastasis from different injection groups was calculated using Adobe Photoshop (version CS5; Adobe Systems, Inc., San Jose, CA, USA). Each dot represented the sum of pixels in the lung metastases in the paraffin-embedded section for each mouse (magnification, x200). Data are representative of at least three separate experiments.
Statistical analysis. Statistical analyses were performed using GraphPad Prism Software (GraphPad Software, Inc., La Jolla, CA, USA). Briefly, data are presented as the mean \pm standard error of the mean or mean \pm standard deviation of three independent experiments. Two-way analysis of variance (ANOVA) followed by Bonferroni's post-hoc test was used to compare the means of groups affected by two independent factors, whereas a one-way ANOVA followed by Tukey's post-hoc test was used to compare the means of three independent groups. Student's t test was used to compare the means of two independent groups. $\mathrm{P}<0.05$ was considered to indicate a statistically significant difference.

Ethics statement. All animal experiments in the present study were approved by Zhejiang University Animal Care Committee (Hangzhou, China). All animal manipulations were performed in accordance with the National Institutes of Health Guide for the Care and Use of Laboratory Animals (NIH, eighth edition), revised in 2012. The mice were sacrificed using carbon dioxide inhalation. All surgery was performed under sodium pentobarbital anesthesia, and all efforts were made by the attending skilled technician to minimize suffering.

\section{Results}

Proliferation of BCap37 cells is not affected by the expression of Bcl-2 in vivo or in vitro. To investigate the effects of Bcl-2 in breast cancer cells in the present study, the expression of Bcl-2 in the BCap37 human breast cancer line was increased or silenced. To increase the expression of Bcl-2, BCap37 cells were transfected either with a $\mathrm{CMV}$ promoter-driven $\mathrm{Bcl}-2$ expression vector (BC-Bcl2 $1 \#$ and $\mathrm{BC}-\mathrm{Bcl} 2$ 2\#) or with an empty vector (BC-Mock). Stable cell lines were established and expanded. To inhibit the expression of Bcl-2, the cells were transduced with a lentiviral vector expressing shRNA-Bcl2 (BC-shRNA-Bcl2 1\# and BC-shRNA-Bcl2 2\#) or with an empty vector (BC-shRNA-NC). The expression level of Bcl-2 in the different cell lines was verified by RT-qPCR and western blot analyses. Bcl-2 was expressed at high levels in the $\mathrm{BC}-\mathrm{Bcl} 2$ cells, compared with its expression in the BC-Mock cells (Fig. 1A and B). Furthermore, Bcl-2 was effectively silenced in the BC-shRNA-Bcl2 cells, when compared with its level in the BC-shRNA-NC cells. BC-Bcl2 2\# and BC-shRNA-Bcl2 $1 \#$ were selected for the following experiments.

To examine the effect of Bcl-2 on the proliferation of BCap37 cells in vitro, the growth rate of BCap37 cells was compared following varying of the expression of Bcl-2. Using the CCK8 assay, no significant differences were observed in the growth rates of the BCap37, BC-Mock, BC-Bcl2, BC-shRNA-NC or BC-shRNA-Bcl2 cells (Fig. 1C).

Subsequently, to assess the effect of $\mathrm{Bcl}-2$ on the proliferation kinetics of BCap37 cells in vivo, the above-mentioned cells were implanted into the right flanks of homozygous nude athymic mice. The tumor volumes following implantation of the different cells were measured every 3 days. After 30 days, when the animals were sacrificed, the mean tumor volumes in the BCap37, BC-Mock, BC-Bcl2, BC-shRNA-NC and BC-shRNA-Bcl2 groups were similar at $\sim 2,059.7 \pm 311.1,2,048.3 \pm 290.6,1,855.5 \pm 447.4,1,898.3 \pm 277.2$ and $1,806.5 \pm 355.1 \mathrm{~mm}^{3}$, respectively $(\mathrm{n}=5$ for each group, 

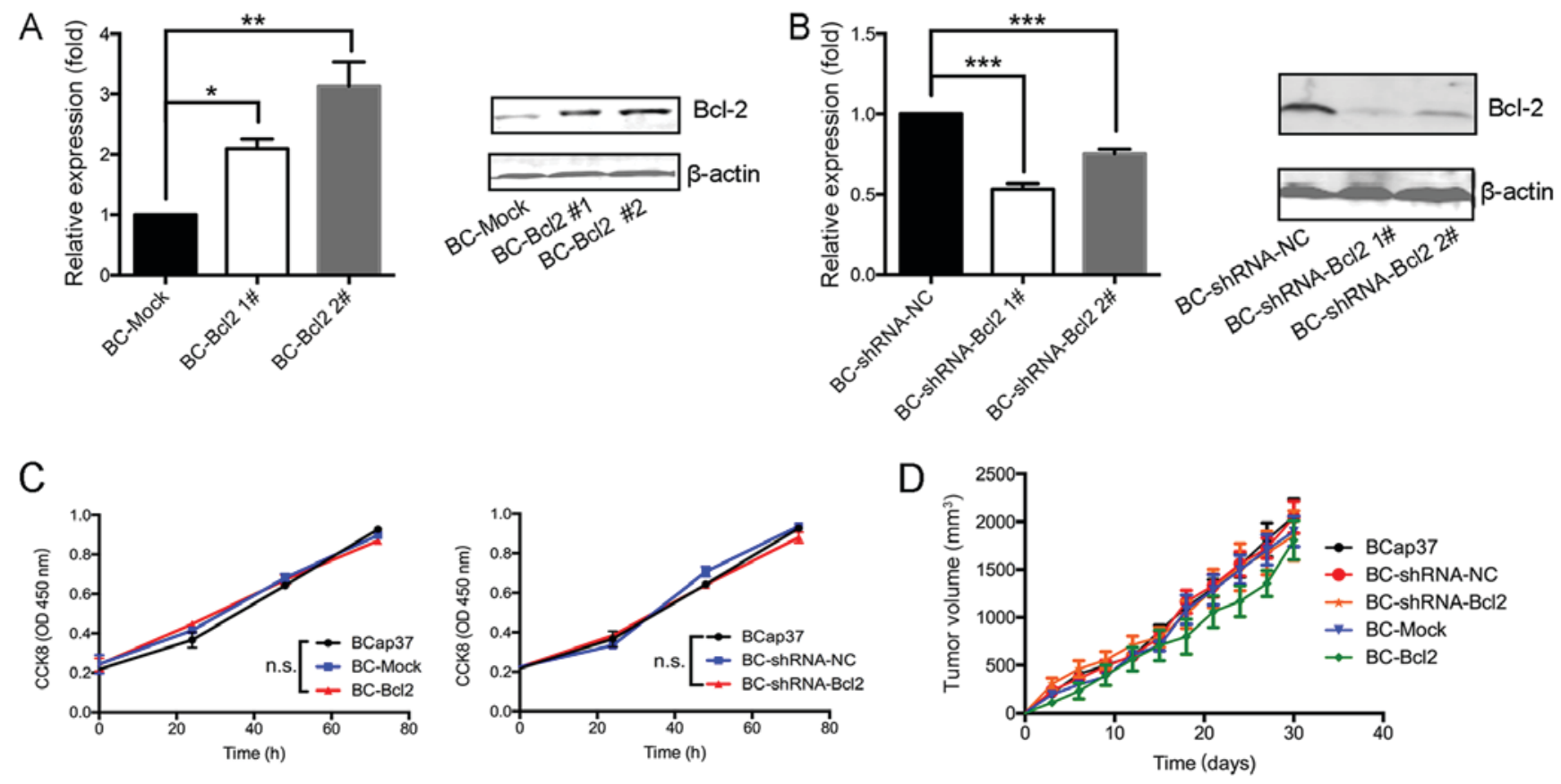

Figure 1. Proliferation of $\mathrm{BCap} 37$ cells is not affected by the expression of $\mathrm{Bcl}-2$ in vivo or in vitro. Results of reverse transcription-quantitative polymerase chain reaction (left) and western blot (right) analyses showed that (A) Bcl-2 was expressed at high levels in BC-Bcl2 cells, compared with its levels in BC-Mock cells, and (B) Bcl-2 was effectively silenced in the BC-shRNA-Bcl2 cells, compared with the BC-shRNA-NC cells. Protein levels were quantitated by densitometry. (C) CCK8 assay revealed no statistically significant difference in relative cellular viability of BCap37 cells upon Bcl-2 overexpression or silencing, compared with the control. (D) Tumor growth was not affected by Bcl-2 in BCap37 tumor xenografts. Nude athymic mice were implanted with BCap37, BC-shRNA-NC, BC-shRNA-Bcl2, BC-Mock, and BC-Bcl2, respectively ( $\mathrm{n}=5$ for each group). The tumor growth curves in each group are shown. The data are presented as the mean \pm standard deviation of three independent experiments. ${ }^{*} \mathrm{P}<0.05,{ }^{* *} \mathrm{P}<0.01,{ }^{* * *} \mathrm{P}<0.001$. BC, BCap37 cell; Bcl-2, B-cell lymphoma 2; shRNA, short hairpin RNA; NC, negative control; CCK8, Cell Counting kit-8; n.s., not significant.

Fig. 1D). There were no significant statistical differences among tumor volumes for the different cell groups. In conclusion, the proliferation of BCap37 cells was not affected by the expression of $\mathrm{Bcl}-2$, neither in vitro nor in vivo.

Bcl-2-overexpressing BCap37 cells exhibit a higher migratory and invasive capacity, compared with control cells in vitro. Subsequently, the present study examined whether Bcl-2 affected the migration and invasion capacity of BCap37 cells. Using a wound-healing assay, it was observed that the inhibition of Bcl-2 in BCap37 cells was associated with the inhibition of wound closure, compared with that in the control. Additionally, the overexpression of Bcl-2 in BCap37 cells accelerated wound closure (Fig. 2A and B). A migration chamber assay was used to verify the role of Bcl-2 in cell motility. Bcl-2 significantly promoted the migration of BCap37 cells, whereas the knockdown of Bcl-2 in BCap37 cells inhibited their migration (Fig. 2C upper panel, and D). The invasion chamber assay showed that Bcl-2 increased the invasive capacity of the BCap37 cells. Taken together, these results suggested that $\mathrm{Bcl}-2$-overexpressing $\mathrm{BCap} 37$ cells exhibit a higher migratory and invasive capacity, compared with control cells in vitro.

Bcl-2 induces lung metastasis of BCap37 cells in vivo. To investigate the effect of $\mathrm{Bcl}-2$ on the kinetics of metastasis of BCap37 cells in vivo, BCap37, BC-Mock, BC-Bc12, BC-shRNA-NC or BC-shRNA-Bcl2 cells were implanted into the right flanks of homozygous nude athymic mice. Following sacrifice of the animals 30 days post-implantation, the lungs were harvested and stained with $\mathrm{H} \& \mathrm{E}$. As shown in Fig. 3A and B, the overexpression of Bcl-2 significantly induced the migration of BCap37 cells to the lung. Therefore, Bcl-2 induced the metastasis of BCap37 cells in vivo.

Overexpression of Bcl-2 in BCap37 cells induces the progression of EMT. To investigate the effect of Bcl-2 on the progression of EMT, a western blot assay was used to detect the protein levels of EMT markers in cells expressing BC-Mock BC-Bcl2, BC-shRNA-NC or BC-shRNA-Bcl2. The western blot results showed that the overexpression of Bcl-2 inhibited the expression of E-cadherin (an epithelial marker), but increased the levels of $\mathrm{N}$-cadherin and vimentin (mesenchymal markers). In addition, the inhibition of Bcl-2 led to an increase in the expression of E-cadherin, but inhibited the expression of $\mathrm{N}$-cadherin and vimentin (Fig. 4). These results are in agreement with previous reports $(18,21)$, suggesting that the overexpression of $\mathrm{Bcl}-2$ increases breast cancer invasion and metastasis by inducing EMT.

\section{Discussion}

$\mathrm{Bcl}-2$ is known as the first proto-oncogene by inhibiting apoptosis, however, the role of $\mathrm{Bcl}-2$ in breast cancer migration and metastasis remains to be fully elucidated. In the present study, it was first found that the proliferation of BCap37 cells was not affected by the expression of $\mathrm{Bcl}-2$, neither in vitro nor in vivo. It has been reported that the knockdown of Bcl-2 has no anti-proliferative effects on colorectal cancer cells (30). Presumably, this may be due to the antagonistic effect of 


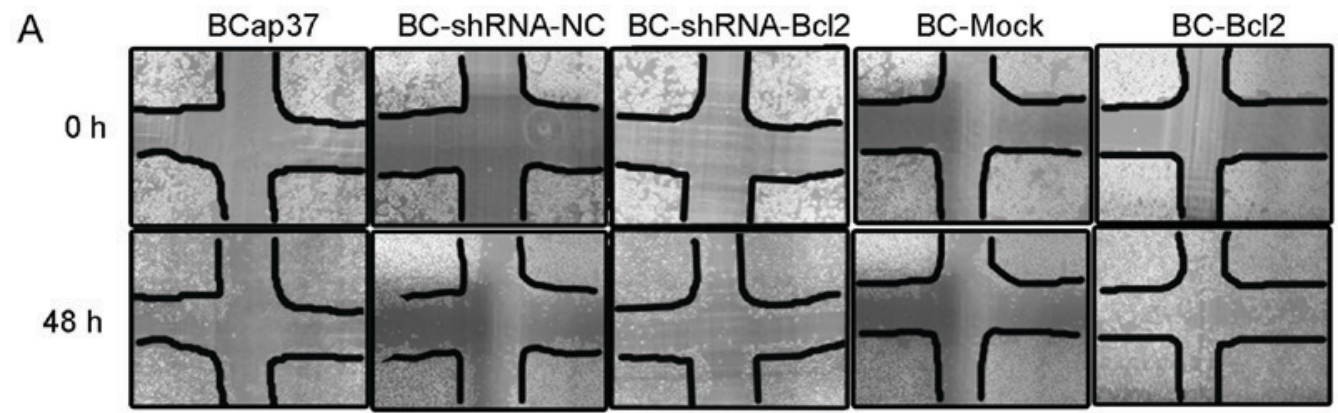

B
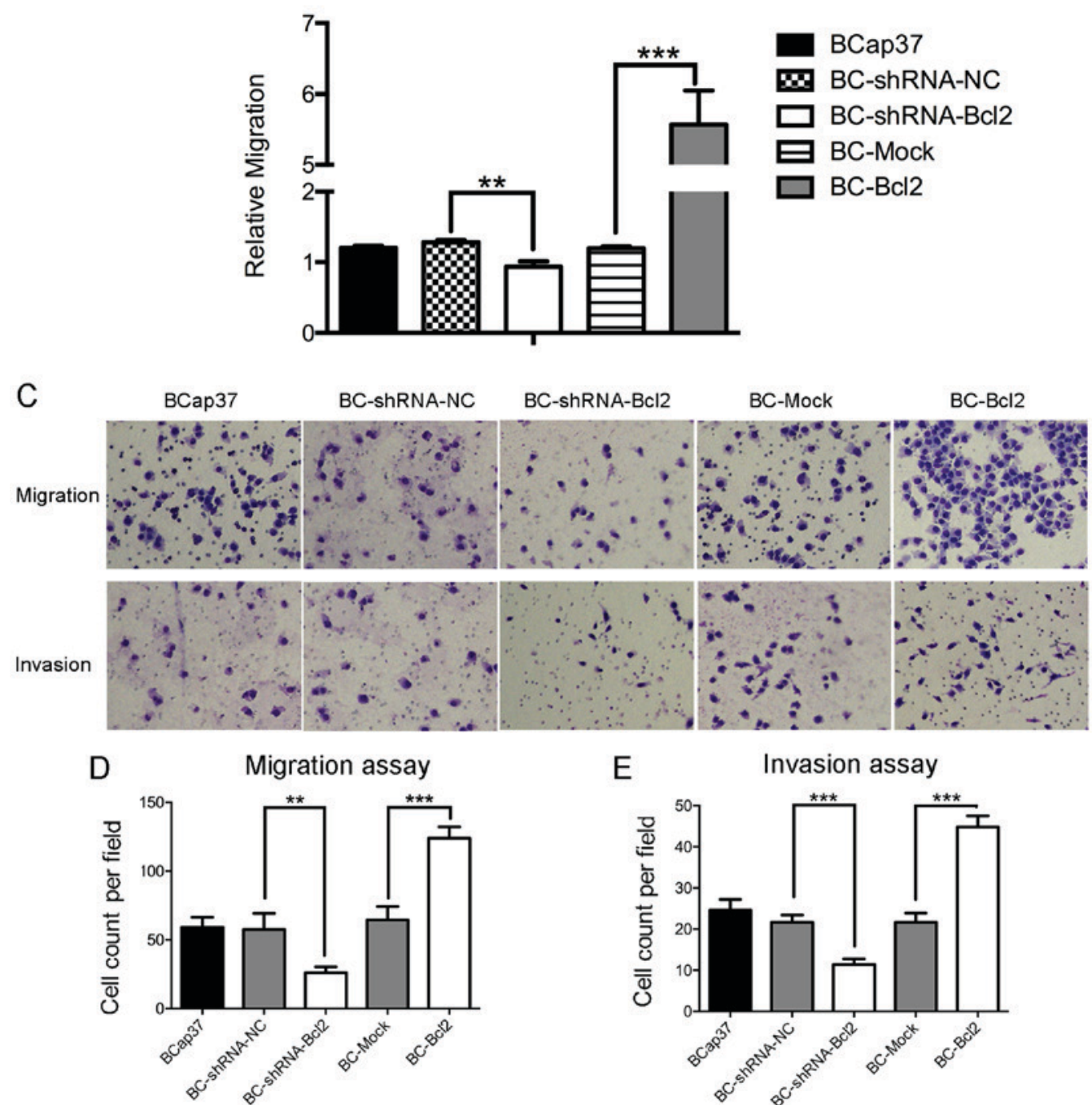

Figure 2. Bcl-2-overexpressing BCap37 cells exhibit a higher migratory and invasive capacity, compared with control cells in vitro. (A) Representative micrographs (magnification, $\mathrm{x} 100$ ) and results of (B) wound-healing assay in cells expressing BC-shRNA-NC, BC-shRNA-Bcl2, BC-Mock and BC-Bcl2, performed with a 48-h recovery period. Relative migration was calculated as follows: (Cell covered areas ( $48 \mathrm{~h}$ )/Cell covered areas $(0 \mathrm{~h})$ ). (C) Representative micrographs of Transwell (D) migration and (E) invasion assays (magnification, x200). Bcl-2 enhanced the migratory and invasive capacity of BCap37 cells in vitro. The data are presented as the mean \pm standard deviation of three independent experiments. ${ }^{* *} \mathrm{P}<0.01,{ }^{* * *} \mathrm{P}<0.001$. BC, BCap37 cell; Bcl-2, B-cell lymphoma 2 ; shRNA, short hairpin RNA; NC, negative control.

other members of the Bcl-2 family, of which there are several members and a number of which have an anti-proliferative function, with others having the opposite effect. For example, $\mathrm{Bcl}-2$-associated $\mathrm{X}$ factor can have negative effects on the anti-apoptotic function of $\mathrm{Bcl}-2$ by forming two dimers with $\mathrm{Bcl}-2$ (31). The present study found that $\mathrm{Bcl}-2$ promoted the migration and metastasis of BCap37 cells in vitro and
Bcl-2-overexpressing BCap37 cells were more likely to transfer to the lungs in mice. In line with previous findings, the present study showed the ability of Bcl-2 to regulate cancer invasion and metastasis, and is the first, to the best of our knowledge, to report its function specifically in breast cancer. EMT is one of the key and common events occur in the early age of cancer development. In the present study, it was observed that Bcl-2 
A

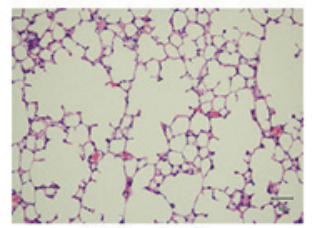

BCap37

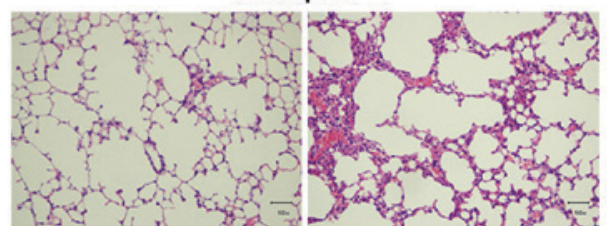

BC-shRNA-NC BC-ShRNA-Bcl2

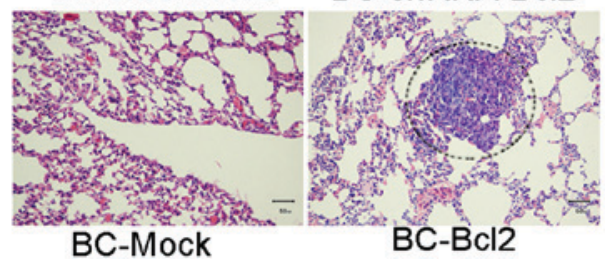

B

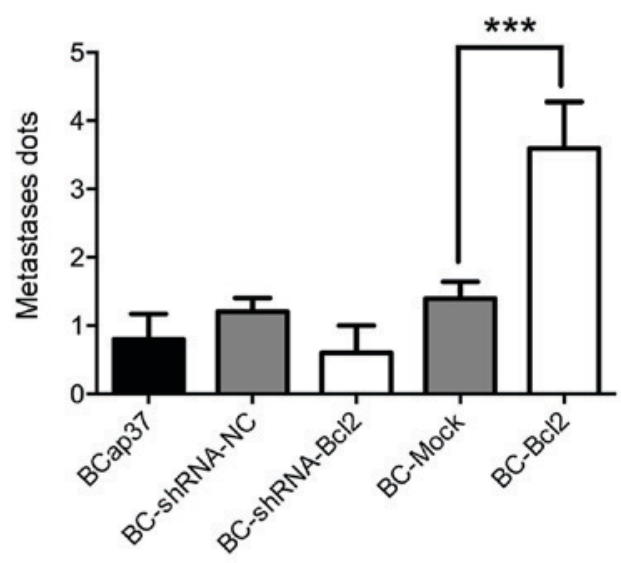

Figure 3. Bcl-2 induces the metastasis of BCap37 cells to the lung in vivo. BCap37, BC-Mock BC-Bcl2, BC-shRNA-NC or BC-shRNA-Bcl2 cells were implanted into the right flanks of homozygous nude athymic mice ( $\mathrm{n}=5$ for each group). (A) Representative micrographs (magnification, $\mathrm{x} 200)$ and (B) results of histopathological analyses of lung tissues from each group, 30 days post-implantation. ${ }^{* * *} \mathrm{P}<0.001$. BC, BCap37 cell; Bcl-2, B-cell lymphoma 2; shRNA, short hairpin RNA; NC, negative control.
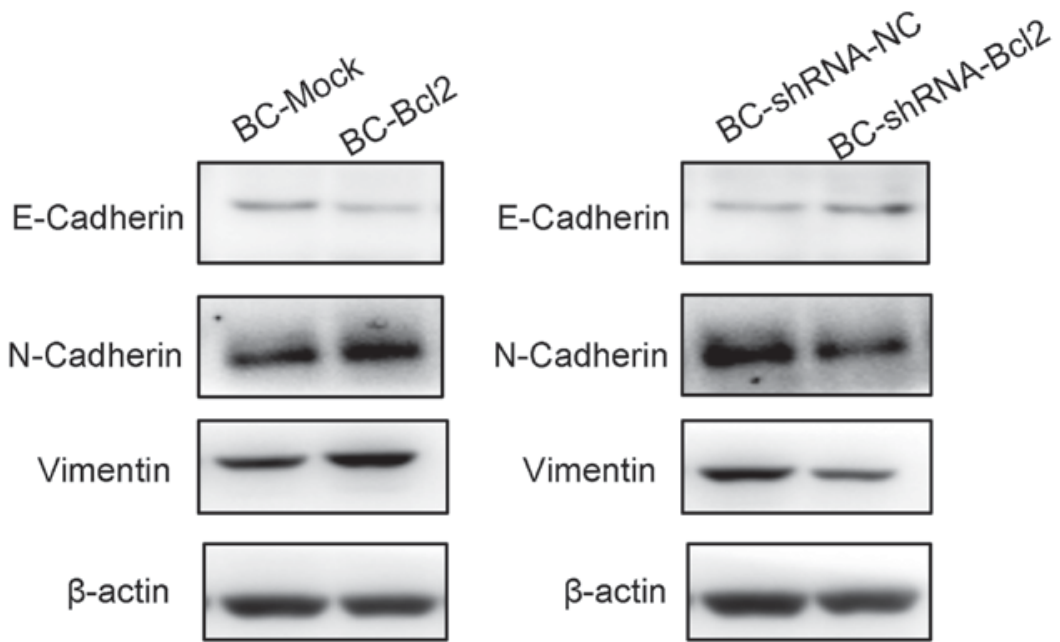

Vimentin

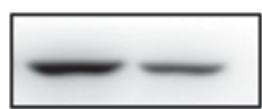

$\beta$-actin

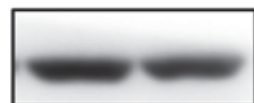

Figure 4. Overexpression of Bcl-2 in BCap37 cells induces the progression of epithelial-to-mesenchymal transition. Western blot assay of cells expressing BC-Mock BC-Bcl2, BC-shRNA-NC or BC-shRNA-Bcl2. Quantification showed that the overexpression of Bcl-2 inhibited the expression of E-cadherin, but increased the levels of $\mathrm{N}$-cadherin and vimentin. The inhibition of Bcl-2 increased the expression of E-cadherin, but inhibited that of $\mathrm{N}$-cadherin and vimentin. Protein levels were quantified by densitometry. BC, BCap37 cell; Bcl-2, B-cell lymphoma 2; shRNA, short hairpin RNA; NC, negative control.

induced EMT progression in BCap37 cells, indicating the early and extensive involvement of Bcl-2 in regulating cancer invasion.

As Bcl-2 is vital in tumorigenesis and neoplastic progression, it may be a promising candidate for anticancer therapy. Previous findings confirmed that Bcl-2 offers potential as a prospective drug target. Several small molecular inhibitors of Bcl-2, including ABT-737 and ABT-199, have been investigated extensively $(16,32)$. ABT-737, designed to neutralize the prosurvival proteins BCL-2, BCL-XL and BCL-W, was reported to improve anti-estrogen tamoxifen susceptibility in the treatment of ER-positive breast cancer and triple negative breast tumor xenografts $(18,33,34)$. In addition, it has been reported that herceptin-resistant breast cancer cells show increased sensitivity to ABT-737, suggesting that ABT-737 offers potential as a therapeutic agent (35). ABT-199 (venetoclax), a potent Bcl-2-selective inhibitor previously approved by the Food and Drug Administration for the treatment of chronic lymphocytic leukemia, was reported to be effective in breast cancer treatment, with satisfactory safety and efficacy $(36,37)$. The combination of Bcl-2/Bcl-xl inhibitor ABT-263/ABT-199 with cyclin-dependent kinase 7 inhibitor thzl can further promote anti-proliferation and induce apoptosis in human triple-negative breast tumor cells (38), showing the compatibility of $\mathrm{Bcl}-2$ chemical inhibitors with other chemotherapeutic drugs. 
In conclusion, the present study comprehensively investigated the effect of Bcl-2 on the motility of the BCap37 cell line. It was found that $\mathrm{Bcl}-2$ did not affect the proliferation of BCap37 cells, but promoted cell invasion in vitro and in vivo. In addition, a lung metastasis mouse model of breast cancer was developed, which may be used to evaluate the effectiveness of certain novel medications for metastatic breast cancer in further investigations. For example, one first-in-class CDK4/6 inhibitor, palbociclib, was approved for the treatment of postmenopausal women with ER(+), HER2(-) advanced breast cancer in combination with letrozole, and achieved a good outcome (39-41). In addition, utidelone, an analog of epothilone, has been tested in a phase II trial as a monotherapy or in combination with capecitabine in patients with heavily pre-treated metastatic breast cancer, and the results showed marked advantages of this novel medication with higher efficacy and tolerability (42). The mouse model established in the present study may be used by others to assess these novel agents in vivo. Upon implantation of breast cancer cells and successful model establishment, these agents can be transferred into the body by intravenous injection to determine their effect on tumor growth featured by the number and size of lung metastases. In addition, the combination of agents and cancer cell lines can vary according to the investigation design. For example, as a supplement, the MCF-7 cell line, which expresses ERs, but not HER2, may be used in the model to assess the effect of palbociclib mentioned above. However, the molecular mechanisms underlying the different effects of $\mathrm{Bcl}-2$ on proliferation, migration and invasion require further investigation.

\section{Acknowledgements}

The authors would like to thank Professor Youfa Zhu, Ms Yanwei Li and Ms Li Liu of the Public Platform of Zhejiang University of Medicine, for providing technical support for the flow cytometry and immunohistochemical assays.

\section{Funding}

No funding was received.

\section{Availability of data and materials}

The analyzed data sets generated during the study are available from the corresponding author, on reasonable request.

\section{Authors' contributions}

PF conceived and designed the experiments. CD performed the majority of the experiments, including the in vivo work, the and in vitro migration and invasion assays, and contributed to the western blotting. XZ, MY and KL performed the CCK-8 assay and analyzed the majority of the data. JW, LC and SW performed RT-qPCR and helped analyze the HE staining.

\section{Ethics approval and consent to participate}

The present study was approved by the Zhejiang University Animal Care Committee (Hangzhou, China). All animal manipulations were performed in accordance with the National Institutes of Health Guide for the Care and Use of Laboratory Animals (8th edition), revised in 2012.

\section{Consent for publication}

Not applicable.

\section{Competing interests}

The authors declare that they have no competing interests.

\section{References}

1. Torre LA, Bray F, Siegel RL, Ferlay J, Lortet-Tieulent J and Jemal A: Global cancer statistics, 2012. CA Cancer J Clin 65: 87-108, 2015

2. Tevaarwerk AJ, Gray RJ, Schneider BP, Smith ML, Wagner LI, Fetting JH, Davidson N, Goldstein LJ, Miller KD and Sparano JA: Survival in patients with metastatic recurrent breast cancer after adjuvant chemotherapy: Little evidence of improvement over the past 30 years. Cancer 119: 1140-1148, 2013.

3. Jiang YL and Liu ZP: Natural products as anti-invasive and anti-metastatic agents. Curr Med Chem 18: 808-829, 2011.

4. Ci Y, Qiao J and Han M: Molecular mechanisms and metabolomics of natural polyphenols interfering with breast cancer metastasis. Molecules 21: E1634, 2016.

5. Borner C: The Bcl-2 protein family: Sensors and checkpoints for life-or-death decisions. Mol Immunol 39: 615-647, 2003.

6. Chipuk JE, Moldoveanu T, Llambi F, Parsons MJ and Green DR: The BCL-2 family reunion. Mol Cell 37: 299-310, 2010.

7. Adams JM and Cory S: Life-or-death decisions by the Bcl-2 protein family. Trends Biochem Sci 26: 61-66, 2001.

8. Um HD: Bcl-2 family proteins as regulators of cancer cell invasion and metastasis: A review focusing on mitochondrial respiration and reactive oxygen species. Oncotarget 7: 5193-5203, 2016.

9. Wick W, Wagner S, Kerkau S, Dichgans J, Tonn JC and Weller M: BCL-2 promotes migration and invasiveness of human glioma cells. FEBS Lett 440: 419-424, 1998.

10. Wick W, Wild-Bode C, Frank B and Weller M: BCL-2-induced glioma cell invasiveness depends on furin-like proteases. J Neurochem 91: 1275-1283, 2004.

11. Choi J, Choi K, Benveniste EN, Rho SB, Hong YS, Lee JH, Kim J and Park K: Bcl-2 promotes invasion and lung metastasis by inducing matrix metalloproteinase-2. Cancer Res 65: 5554-5560, 2005.

12. Del Bufalo D, Biroccio A, Leonetti C and Zupi G: Bcl-2 overexpression enhances the metastatic potential of a human breast cancer line. FASEB J 11: 947-953, 1997.

13. An J, Lv J, Li A, Qiao J, Fang L, Li Z, Li B, Zhao W, Chen H and Wang L: Constitutive expression of Bcl-2 induces epithelial-Mesenchymal transition in mammary epithelial cells. BMC Cancer 15: 476, 2015.

14. Kang Y, Siegel PM, Shu W, Drobnjak M, Kakonen SM, Cordón-Cardo C, Guise TA and Massagué J: A multigenic program mediating breast cancer metastasis to bone. Cancer Cell 3: 537-549, 2003.

15. Pinkas J, Martin SS and Leder P: Bcl-2-mediated cell survival promotes metastasis of EpH4 betaMEKDD mammary epithelial cells. Mol Cancer Res 2: 551-556, 2004.

16. Cang S, Iragavarapu C, Savooji J, Song Y and Liu D: ABT-199 (venetoclax) and BCL-2 inhibitors in clinical development. J Hematol Oncol 8: 129, 2015.

17. Dawson SJ, Makretsov N, Blows FM, Driver KE, Provenzano E, Le Quesne J, Baglietto L, Severi G, Giles GG, McLean CA, et al: BCL2 in breast cancer: A favourable prognostic marker across molecular subtypes and independent of adjuvant therapy received. Br J Cancer 103: 668-675, 2010.

18. Oakes SR, Vaillant F, Lim E, Lee L, Breslin K, Feleppa F, Deb S, Ritchie ME, Takano E, Ward T, et al: Sensitization of BCL-2-expressing breast tumors to chemotherapy by the BH3 mimetic ABT-737. Proc Natl Acad Sci USA 109: 2766-2771, 2012.

19. Kerr DA II and Wittliff JL: A five-gene model predicts clinical outcome in ER+/PR+, early-stage breast cancers treated with adjuvant tamoxifen. Horm Cancer 2: 261-271, 2011. 
20. Choi JE, Kang SH, Lee SJ and Bae YK: Prognostic significance of $\mathrm{Bcl}-2$ expression in non-basal triple-negative breast cancer patients treated with anthracycline-based chemotherapy. Tumour Biol 35: 12255-12263, 2014.

21. Neri A, Marrelli D, Roviello F, DeMarco G, Mariani F, DeStefano A, Megha T, Caruso S, Corso G, Cioppa T and Pinto E: Bcl-2 expression correlates with lymphovascular invasion and long-term prognosis in breast cancer. Breast Cancer Res Treat 99: 77-83, 2006.

22. Ishijima N, Miki C, Ishida T, Kinoshita $T$ and Suzuki H: The immunohistochemical expression of BCL-2 oncoprotein in colorectal adenocarcinoma. Surg Today 29: 682-684, 1999.

23. Lin D, Kuang G, Wan J, Zhang X, Li H, Gong X and Li H: Luteolin suppresses the metastasis of triple-negative breast cancer by reversing epithelial-to-mesenchymal transition via downregulation of $\beta$-catenin expression. Oncol Rep 37: 895-902, 2017.

24. Hazan RB, Phillips GR, Qiao RF, Norton L and Aaronson SA: Exogenous expression of $\mathrm{N}$-cadherin in breast cancer cells induces cell migration, invasion, and metastasis. J Cell Biol 148: $779-790,2000$

25. Sun T, Sun BC, Zhao XL, Zhao N, Dong XY, Che N, Yao Z, Ma YM, Gu Q, Zong WK and Liu ZY: Promotion of tumor cell metastasis and vasculogenic mimicry by way of transcription coactivation by Bcl-2 and Twist1: A study of hepatocellular carcinoma. Hepatology 54: 1690-1706, 2011.

26. Zuo J, Ishikawa T, Boutros S, Xiao Z, Humtsoe JO and Kramer RH: Bcl-2 overexpression induces a partial epithelial to mesenchymal transition and promotes squamous carcinoma cell invasion and metastasis. Mol Cancer Res 8: 170-182, 2010.

27. Lu PJ, Lu QL, Rughetti A and Taylor-Papadimitriou J: Bcl-2 overexpression inhibits cell death and promotes the morphogenesis, but not tumorigenesis of human mammary epithelial cells J Cell Biol 129: 1363-1378, 1995.

28. Jiang D, Sui M, Zhong W, Huang Y and Fan W: Different administration strategies with paclitaxel induce distinct phenotypes of multidrug resistance in breast cancer cells. Cancer Lett 335: 404-411, 2013.

29. Livak KJ and Schmittgen TD: Analysis of relative gene expression data using real-time quantitative PCR and the 2(-Delta Delta C(T)) method. Methods 25: 402-408, 2001

30. Koehler BC, Scherr AL, Lorenz S, Urbanik T, Kautz N, Elssner C, Welte S, Bermejo JL, Jäger D and Schulze-Bergkamen $H$ Beyond cell death-antiapoptotic $\mathrm{Bcl}-2$ proteins regulate migration and invasion of colorectal cancer cells in vitro. PLoS One 8 e76446, 2013.

31. Bonnefoy-Berard N, Aouacheria A, Verschelde C, Quemeneur L, Marçais A and Marvel J: Control of proliferation by Bcl-2 family members. Biochim Biophys Acta 1644: 159-168, 2004.
32. Pan R, Ruvolo VR, Wei J, Konopleva M, Reed JC, Pellecchia M, Andreeff $M$ and Ruvolo PP: Inhibition of Mcl-1 with the pan-Bcl-2 family inhibitor (-)BI97D6 overcomes ABT-737 resistance in acute myeloid leukemia. Blood 126: 363-372, 2015.

33. Oltersdorf T, Elmore SW, Shoemaker AR, Armstrong RC, Augeri DJ, Belli BA, Bruncko M, Deckwerth TL, Dinges J, Hajduk PJ, et al: An inhibitor of Bcl-2 family proteins induces regression of solid tumours. Nature 435: 677-681, 2005.

34. Chen J, Jin S, Abraham V, Huang X, Liu B, Mitten MJ, Nimmer P, Lin X, Smith M, Shen Y, et al: The Bcl-2/Bcl-X(L)/Bcl-w inhibitor, navitoclax, enhances the activity of chemotherapeutic agents in vitro and in vivo. Mol Cancer Ther 10: 2340-2349, 2011.

35. Crawford A and Nahta R: Targeting Bcl-2 in herceptin-resistant breast cancer cell lines. Curr Pharmacogenomics Persona Med 9: 184-190, 2011.

36. Vaillant F, Merino D, Lee L, Breslin K, Pal B, Ritchie ME, Smyth GK, Christie M, Phillipson LJ, Burns CJ, et al: Targeting BCL-2 with the BH3 mimetic ABT-199 in estrogen receptor-positive breast cancer. Cancer Cell 24: 120-129, 2013.

37. Deng J and Letai A: Priming BCL-2 to kill: The combination therapy of tamoxifen and ABT-199 in ER+ breast cancer. Breast Cancer Res 15: 317, 2013.

38. Li B, Ni Chonghaile T, Fan Y, Madden SF, Klinger R, O'Connor AE, Walsh L, O'Hurley G, Mallya Udupi G, Joseph $\mathrm{J}$, et al: Therapeutic rationale to target highly expressed CDK7 conferring poor outcomes in triple-negative breast cancer. Cancer Res 77: 3834-3845, 2017.

39. Dukelow T, Kishan D, Khasraw M and Murphy CG: CDK4/6 inhibitors in breast cancer. Anticancer Drugs 26: 797-806, 2015.

40. Xu H, Yu S, Liu Q, Yuan X, Mani S, Pestell RG and Wu K: Recent advances of highly selective CDK4/6 inhibitors in breast cancer. J Hematol Oncol 10: 97, 2017.

41. Lu J: Palbociclib: A first-in-class CDK4/CDK6 inhibitor for the treatment of hormone-receptor positive advanced breast cancer. J Hematol Oncol 8: 98, 2015.

42. Zhang $\mathrm{P}$, Tong Z, Tian F, Wang Y, Yang J, Li W, Di L, Liu W, Tang L, Qiu R and Xu B: Phase II trial of utidelone as monotherapy or in combination with capecitabine in heavily pretreated metastatic breast cancer patients. J Hematol Oncol 9: 68, 2016.

This work is licensed under a Creative Commons Attribution-NonCommercial-NoDerivatives 4.0 International (CC BY-NC-ND 4.0) License. 